\title{
Significant Association Between Coronary Artery Low-Attenuation Plaque Volume and Apnea-Hypopnea Index, But Not Muscle Sympathetic Nerve Activity, in Patients With Obstructive Sleep Apnea Syndrome
}

\author{
Takuto Hamaoka, MD, PhD; Hisayoshi Murai, MD, PhD; Shuichi Kaneko, MD, PhD; \\ Soichiro Usui, MD, PhD; Oto Inoue, MD; Hiroyuki Sugimoto, MD; Yusuke Mukai, MD; \\ Yoshitaka Okabe, MD; Hideki Tokuhisa, MD, PhD; Shinichiro Takashima, MD, PhD; \\ Takeshi Kato, MD, PhD; Hiroshi Furusho, MD, PhD; Soichiro Kashiwaya; Yu Sugiyama, MD; \\ Yasuto Nakatsumi, MD, PhD; Shigeo Takata, MD, PhD; Masayuki Takamura, MD, PhD
}

\begin{abstract}
Background: Obstructive sleep apnea syndrome (OSAS) is associated with augmented sympathetic nerve activity and cardiovascular diseases. However, the interaction between coronary artery plaque characteristics and sympathetic nerve activity remains unclear. The purpose of this study was to clarify the relationships between coronary artery plaque characteristics, sleep parameters and single- and multi-unit muscle sympathetic nerve activity (MSNA) in OSAS patients.
\end{abstract}

\begin{abstract}
Methods and Results: A total of 32 OSAS patients who underwent full-polysomnography participated in this study. The coronary plaque volume was calculated with 320-slice coronary computed tomography (CT). Single- and multi-unit MSNA were obtained during the daytime within 1 week from full-polysomnography. Patients were divided into 2 groups according to their apnea-hypopnea index $(\mathrm{AHI})$ score (mild-moderate group, $\mathrm{AHI}<30$; and severe group, $\mathrm{AHI} \geq 30$ ). There were no group differences in risk factors for atherosclerosis; however, severe $\mathrm{AHI}$ patients showed significantly high single-unit MSNA, and low- and intermediate-attenuation plaque volumes. In regression analysis, the plaque volume of any CT value was not associated with single- or multi-unit MSNA; only AHI significantly correlated with low-attenuation plaque volume $(R=0.52, P<0.05)$.
\end{abstract}

Conclusions: Our findings provided the evidence that $\mathrm{AHI}$ is an independent predictor for low-attenuated, vulnerable plaque volume, but not daytime MSNA, in patients with OSAS.

Key Words: Apnea-hypopnea index; Coronary computed tomography; Muscle sympathetic nerve activity; Single-unit recordings; Sleep apnea syndrome

$\mathbf{O}$ bstructive sleep apnea syndrome (OSAS) is an independent risk factor for cardiovascular disease (CVD). ${ }^{1,2}$ We recently demonstrated that OSAS severity according to the apnea-hypopnea index (AHI) was more markedly associated with single-unit muscle sympathetic nerve activity (MSNA) compared with multiunit MSNA. ${ }^{3}$ Augmented SNA is believed to play a crucial role in the development of CVD in OSAS patients. ${ }^{4,5}$ According to a previous animal study, SNA increases plaque proliferation by stimulating hematopoietic stem cells, leading to increased output of neutrophils and inflammatory monocytes. ${ }^{6}$ However, the association between the progression of plaque burden in coronary arteries and
SNA in OSAS patients remains unclear.

Coronary computed tomography (CCT) is widely used to assess coronary artery plaque because of its high accuracy and minimally invasive properties. A previous report showed that a higher AHI score is the main contributor to cardiovascular events, ${ }^{2}$ and a significant relationship between the AHI and mean coronary plaque volume evaluated by 64-slice CCT in patients with OSAS has been demonstrated. ${ }^{7}$ The recently developed 320 -slice CCT has the potential to evaluate coronary arteries with high image quality, regardless of the patient's heartbeat, ${ }^{8}$ which could clarify not only plaque volume and also characteristics in more detail compared with conventional CCT. ${ }^{9}$

Received February 27, 2018; revised manuscript received July 23, 2018; accepted August 2, 2018; released online September 7, 2018 Time for primary review: 15 days

System Biology, Graduate School of Advanced Preventive Medical Sciences, Kanazawa University, Kanazawa (T.H., H.M., S. Kaneko, S.U., O.I., H.S., Y.M., Y.O., H.T., S. Takashima, T.K., H.F., M.T.); Kanazawa Municipal Hospital, Kanazawa (S. Kashiwaya, Y.S., Y.N., S. Takata), Japan

Mailing address: Hisayoshi Murai, MD, PhD, System Biology, Graduate School of Advanced Preventive Medical Science, Kanazawa University, 13-1 Takara-machi, Kanazawa 920-8641, Japan. E-mail: sakurasoma1209@yahoo.co.jp

ISSN-1346-9843 All rights are reserved to the Japanese Circulation Society. For permissions, please e-mail: cj@j-circ.or.jp 


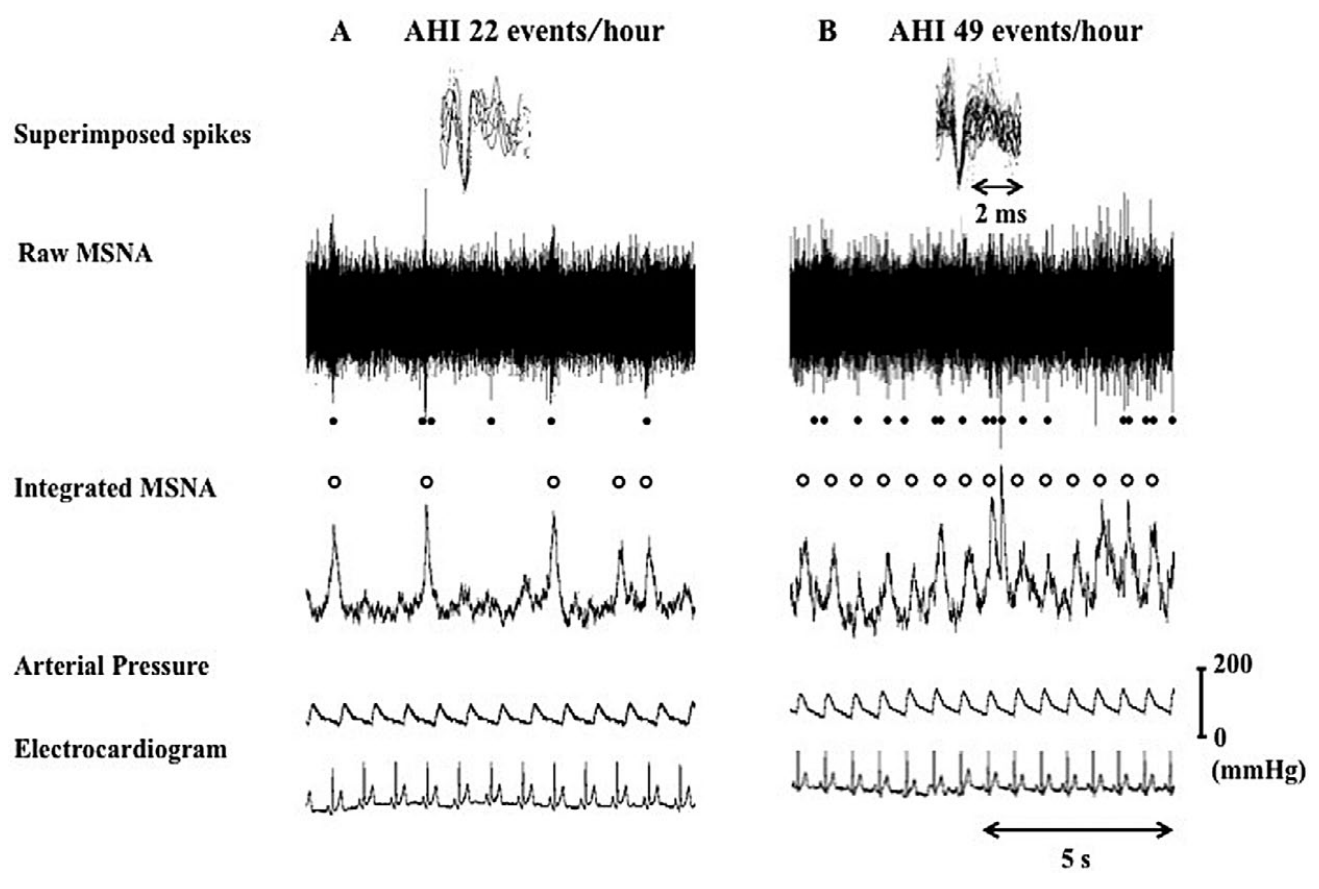

Figure 1. Typical recordings of single- and multi-unit MSNA in 2 patients with OSAS divided by AHI. (A) MSNA recording of one patient with moderate OSAS; (B) MSNA recording of one patient with severe OSAS. Black circles indicate single-unit MSNAs and white circles indicate multi-unit MSNAs. Single-unit MSNAs were confirmed by superimposing the action potentials. AHI, apnea-hypopnea index; MSNA, muscle sympathetic nerve activity; OSAS, obstructive sleep apnea syndrome.

We hypothesized that augmented SNA is related to coronary artery plaque volume and/or characteristics in OSAS patients. To test this hypothesis, we used 320-slice CCT to evaluate the relationship between coronary plaque characteristics and daytime SNA, as assessed by direct recording of MSNA, in patients with OSAS.

\section{Methods}

\section{Subjects}

Patients with OSAS prior to treatment were included in this study. OSAS was diagnosed when the patient's AHI score was $\geq 15$ events $/ \mathrm{h}$ or $\geq 5$ events $/ \mathrm{h}$ with daytime sleepiness. Patients who experienced $\geq 5$ central sleep apnea events/h were excluded, as were patients who had unstable angina pectoris, heart failure, an estimated glomerular filtration rate of $<30 \mathrm{~mL} / \mathrm{min} / 1.73 \mathrm{~m}^{2}$, implanted pacemaker device, or myocardial infarction and/or coronary revascularization within 4 weeks of the study, and current or ever smokers. Heart failure was defined in accordance with the American Heart Association/American College of Cardiology guidelines. ${ }^{10}$ In addition, patients who had uncontrollable risk factors for atherosclerosis despite adequate medication were excluded (systolic blood pressure $>160 \mathrm{mmHg}$, diastolic blood pressure $>100 \mathrm{mmHg}, \mathrm{HbAlc}>7 \%$, low-density lipoprotein (LDL) cholesterol $>160 \mathrm{mg} / \mathrm{dL}$ ). This study was designed as a cross-sectional, observational trial. All investigators assessing MSNA and polysomnography (PSG) data were blinded to the patients' characteristics. The study protocol was approved by the Research Ethics Board of Kanazawa University (Kanazawa, Japan). The study has been registered in the University Hospital Medical Information Network Center (UMIN, TOKYO, Japan) Clinical Trials Registration System as UMIN000017612. All patients provided informed consent.

\section{PSG}

PSG monitoring was performed overnight in the Sleep Disorders Laboratory of Kanazawa Municipal Hospital using an Embla N7000 system (Natus, San Carlos, CA, USA) in accordance with a protocol reported previously. ${ }^{3}$ Briefly, patients were admitted to the laboratory in the evening, and preparation for PSG started at 20:00 hours. The measurements started at 21:00 hours after lights were turned off and continued until the next morning. During the session, electroencephalogram, right and left electrooculogram, body position, thoracic and abdominal wall motion, electrocardiogram, nasal airflow, oxygen saturation, and the patient's sleep state were recorded. These data were subsequently analyzed by experienced investigators. All PSG recordings were administered by a physician registered as a PSG technologist by the American Academy of Sleep Medicine, and a physician certified in sleep medicine by the Japanese Society of Sleep Research. This examination method is included in the type 1 category of the American Academy of Sleep Medicine, American Thoracic Society and the American College of Chest Physicians manuals for the examination of suspected sleep apnea in adults. ${ }^{11}$

\section{Sympathetic Nerve Data Measurement and Analysis}

Postganglionic MSNA was recorded directly from the peroneal nerve as described previously. ${ }^{3}$ In brief, subjects 


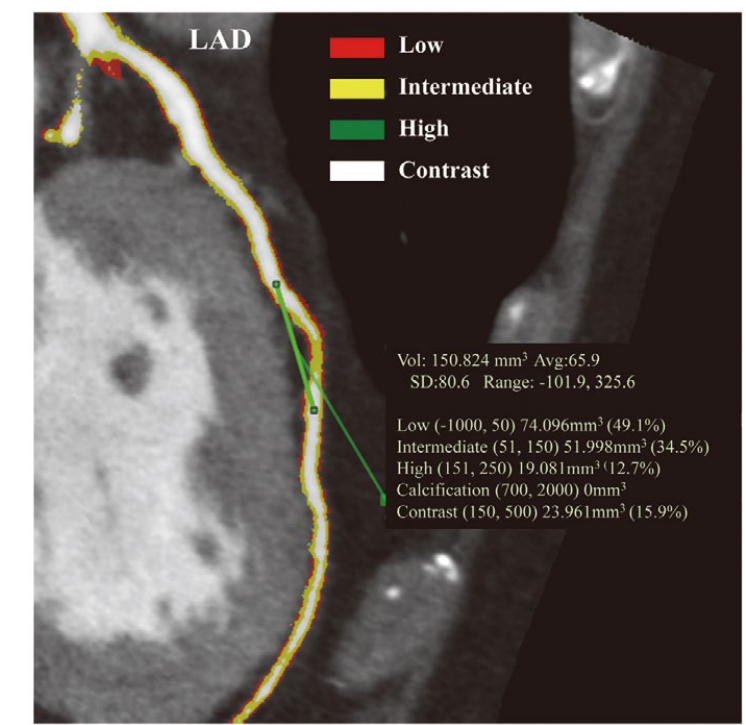

Figure 2. Typical image of plaque volume evaluation in LAD stenosis lesion. Plaque colored red is low-attenuation plaque $(-1,000-50 \mathrm{HU})$, plaque colored yellow is intermediateattenuation plaque $(51-150 \mathrm{HU})$, and plaque colored green is high-attenuation plaque $(151-250 \mathrm{HU})$. Contrast medium is colored white (150-500 HU). Plaque volume included in the vessel area connected by the straight green line was calculated. LAD, left anterior descending coronary artery.

abstained from alcohol and caffeine for $24 \mathrm{~h}$, and at least $12 \mathrm{~h}$ post-prandial MSNA was measured in the spinal position during a morning within 1 week of the PSG evaluation. The common peroneal nerve was identified by palpitation and/or stimulated electrically at the skin surface. A high-impedance $(10 \mathrm{M} \Omega)$ tungsten microelectrode was inserted percutaneously into a motor fascicle of the peroneal nerve and adjusted until a pulse-synchronous large unitary spike was distinguishable from background noise to permit single-unit MSNA analysis. The signals were amplified by a factor of 50,000-100,000, band-pass filtered (500$3,000 \mathrm{~Hz}$ ), and had a resistance-capacitance integrated circuit with a time constant of 0.1 s to produce a mean voltage neurogram using a Power Lab recording system (Model ML 785/85P; ADI Instruments, Bella Vista, NSW, Australia). The raw nerve signal was obtained at $12 \mathrm{kHz}$ and other signals were obtained at $1,000 \mathrm{~Hz}$. MSNA bursts were detected by visual inspection by an experienced investigator blinded to the study protocol. Multi-unit MSNA was expressed as the number of bursts/min (burst frequency) and the number of bursts/100 heartbeats (burst incidence). Additionally, when the raw neurogram record was distinct enough to identify single-unit MSNA spikes, spike morphology was discreetly inspected by an experienced investigator. Single-unit MSNA spikes were defined as (1) spike synchronization with multi-unit MSNA bursts, (2) triphasic spike morphology with a negative main phase, and (3) superimposition of candidate action potentials with minimal variation. Single-unit MSNA was expressed as number/min (spike frequency) and the number/100 heartbeats (spike incidence). Figure 1 presents typical recordings of singleand multi-unit MSNA in moderate and severe OSAS patients.

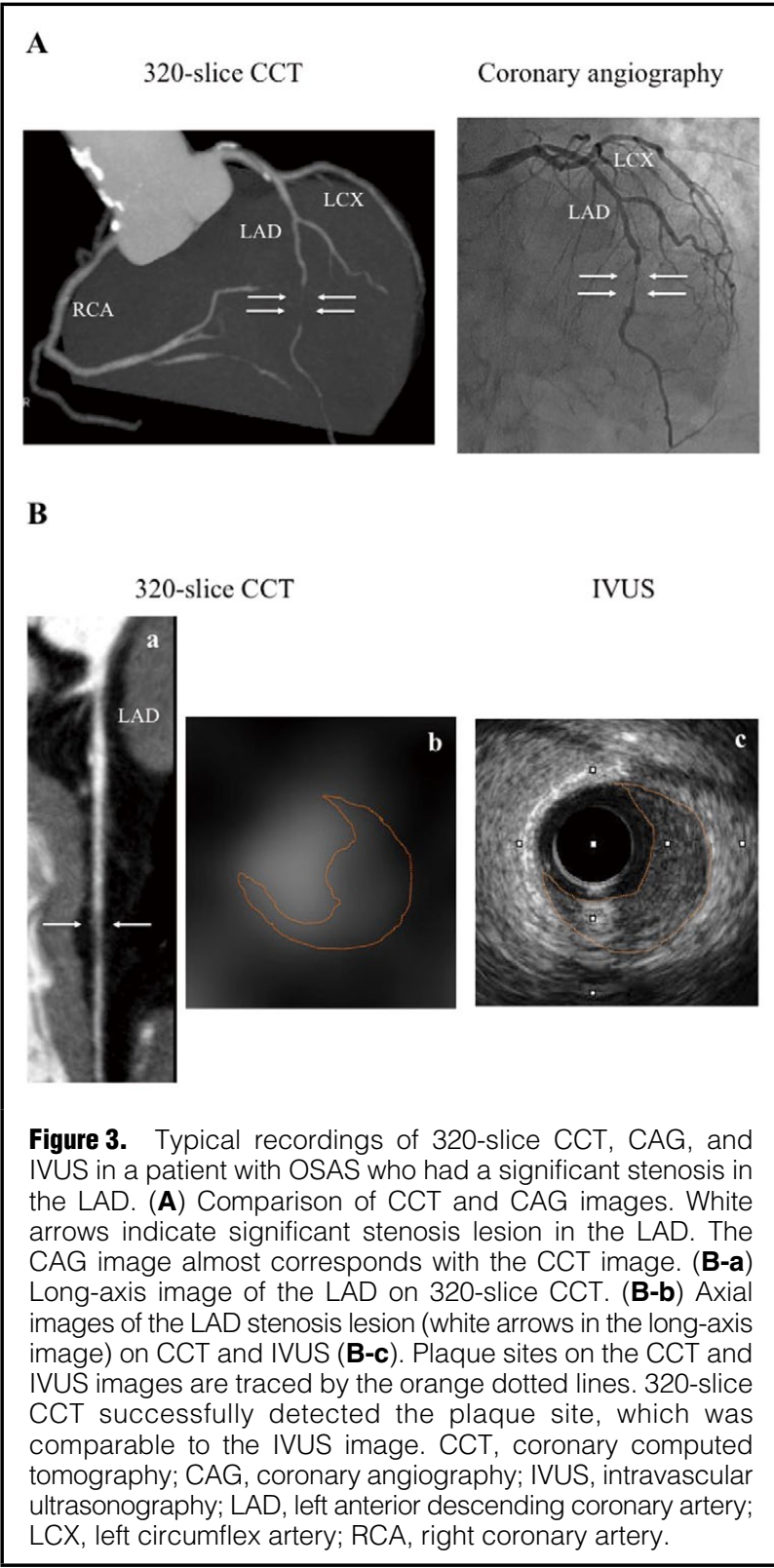

\section{0-Slice CCT}

ECG-gated 320-slice CCT with a slice thickness of $0.5 \mathrm{~mm}$, tube voltage of $120 \mathrm{kV}$, and tube maximum current of $580 \mathrm{~mA}$ was used in this study (Aquilion One, Toshiba Medical Systems, Tokyo, Japan). CCT measurements were performed within 1 month of the PSG evaluation. Contrast medium was injected from the right antecubital vein using a 20-22-gauge needle. After the patient's position was decided and the route of administration was prepared, breath-holding practice was performed. In this practice, the alteration of the patient's heartbeat was carefully evaluated; if the mean heart rate (HR) was $>63$ beats $/ \mathrm{min}$ and the patient had no contraindication for using a $\beta$-adrenergic receptor antagonist, such as for bronchial asthma or symptomatic heart failure, $0.125 \mathrm{mg} / \mathrm{kg}$ of short-acting adrenergic $\beta$-1 blocker (landiolol hydrochloride) was administered, and breath-holding was practiced again. The region of interest (ROI) was placed on the ascending aorta, and 


\begin{tabular}{|lc|}
\hline \multicolumn{2}{|l|}{ Table 1. Baseline Characteristics of Patients With } \\
Obstructive Sleep Apnea \\
Clinical characteristics \\
Age (years) \\
Female/male & $63.3 \pm 10.8$ \\
BMI (kg/m²) & $6 / 26$ \\
Hypertension ( $\mathrm{n}(\%))$ & $26.3 \pm 5.1$ \\
Diabetes mellitus ( $\mathrm{n}(\%))$ & $14(44)$ \\
Dyslipidemia (n (\%)) & $6(19)$ \\
Burst frequency (bursts/min) & $8(25)$ \\
Burst incidence (bursts/100 heartbeats) & $55.5 \pm 12.1$ \\
Spike frequency (spikes/min) & $81.4 \pm 14.6$ \\
Spike Incidence (spikes/100 heartbeats) & $59.0 \pm 12.7$ \\
Systolic BP (mmHg) & $87.8 \pm 17.6$ \\
Diastolic BP (mmHg) & $130.4 \pm 12.9$ \\
Heart rate (beats/min) & $78.8 \pm 11.9$ \\
LDL-cholesterol (mg/dL) & $70.7 \pm 9.7$ \\
HbA1c (\%) & $119.3 \pm 28.6$ \\
Sleep parameters & $5.9 \pm 0.5$ \\
AHI (events/h) & \\
3\%ODI (events/h) & $33.7 \pm 16.1$ \\
Arousal index (events/h) & $26.8 \pm 18.3$ \\
Medications & $33.8 \pm 17.5$ \\
Calcium-channel blocker & \\
ARB or ACEl & $8(25)$ \\
$\beta$-blocker & $9(28.1)$ \\
Diuretic & $1(3.1)$ \\
Statin & $0(0)$ \\
Values are mean \pm SD. ACEl angioten & $7(21.9)$ \\
\hline
\end{tabular}

Values are mean $\pm \mathrm{SD}$. ACEI, angiotensin-converting enzyme inhibitor; AHI, apnea-hypopnea index; ARB, angiotensin II receptor blocker; BMI, body mass index; BP, blood pressure; LDL, lowdensity lipoprotein; ODI, oxygen desaturation index.

recording began (bolus tracking method) when the CT value of the ROI was $>200 \mathrm{HU}$ after administration of the contrast medium. Contrast medium was administered at a dose of $26 \mathrm{mgI} / \mathrm{kg} / \mathrm{s}$ over $12 \mathrm{~s}$ using a syringe injector with a dual flow option (Dual shot GX, Nemoto, Japan). In this study, the HEARTNAVI ${ }^{\circledR}$ system (Toshiba Medical Systems) was used because it provides the best temporal resolution and can be adjusted based on the HR during scanning. The system protocol was described previously. ${ }^{12}$ Briefly, HR was estimated during scanning based on HR during breath holding, and the scanning condition was decided as follows in accordance with the estimated HR: estimated HR $<66$ (beats/min), 1 heartbeat of data was extracted and half reconstructed; estimated HR was 66-79, 2 heartbeats of data were extracted and 2 sector reconstructions were carried out; estimated HR was 80-117, 3 heartbeats of data were extracted and 3 sector reconstruction was carried out; estimated HR was 118-155, 4 heartbeats of data were extracted, and 4 sector reconstruction was carried out; estimated HR was $>155,5$ heartbeats of data were extracted, and 5 sector reconstruction was carried out. If unexpected heartbeats occurred during actual recording and/or HR during the breath-holding practice fluctuated with arrhythmias, such as premature beats or atrial fibrillation, 1 extra heartbeat of data was scanned. After finishing the entire recording, the reconstructed images were transferred to the workstation (ZIOSTATION Z820, Amin, Tokyo, Japan).

\begin{tabular}{|c|c|c|}
\hline & $\underset{(n=15)}{M M}$ & $\underset{(n=17)}{S}$ \\
\hline Age (years) & $61.3 \pm 12.4$ & $65.0 \pm 9.2$ \\
\hline Male (n (\%)) & $11(73.3)$ & $15(88.2)$ \\
\hline $\mathrm{BMI}\left(\mathrm{kg} / \mathrm{m}^{2}\right)$ & $26.3 \pm 5.0$ & $26.2 \pm 5.3$ \\
\hline Systolic BP (mmHg) & $130 \pm 14.7$ & $131 \pm 11.6$ \\
\hline Diastolic BP $(\mathrm{mmHg})$ & $81.6 \pm 11.8$ & $76.2 \pm 11.8$ \\
\hline Heart rate (beats/min) & $72.6 \pm 11.5$ & $68.9 \pm 7.8$ \\
\hline LDL-cholesterol (mg/dL) & $120 \pm 30.9$ & $118 \pm 27.4$ \\
\hline $\mathrm{HbA1c}(\%)$ & $5.9 \pm 0.53$ & $5.9 \pm 0.55$ \\
\hline Hypertension (n (\%)) & $5(30.0)$ & $9(52.9)$ \\
\hline Diabetes mellitus ( $\mathrm{n}(\%)$ ) & $2(13.3)$ & $4(23.5)$ \\
\hline Dyslipidemia (n (\%)) & $3(20.0)$ & $5(29.4)$ \\
\hline $\mathrm{AHI}$ (events/h) & $20.7 \pm 8.4$ & $45.2 \pm 11.9^{*}$ \\
\hline $3 \% O D I$ (events/h) & $15.7 \pm 8.2$ & $36.5 \pm 19.3^{*}$ \\
\hline Arousal index (events/hour) & $25.6 \pm 7.1$ & $40.9 \pm 20.8^{*}$ \\
\hline \multicolumn{3}{|l|}{ Medications } \\
\hline$\beta$-blocker $(\mathrm{n}(\%))$ & $0(0)$ & $1(5.9)$ \\
\hline Calcium-channel blocker & $2(13.3)$ & $6(35.3)$ \\
\hline ACEI or ARB & $3(20.0)$ & $6(35.3)$ \\
\hline Diuretic & $0(0)$ & $0(0)$ \\
\hline Statin & $2(13.3)$ & $5(29.4)$ \\
\hline
\end{tabular}

Values are mean $\pm S D$. ${ }^{*} \mathrm{P}<0.05$. MM, mild-moderate [severity] group; S, severe group. Other abbreviations as in Table 1.

\section{CT Data Analysis}

The CCT images were evaluated using dedicated software (Coronary Artery Analysis 2, Amin) by an experienced cardiologist. The severity of stenosis and plaque characteristics was carefully evaluated in the original axial dataset and curved planar reformation images. Coronary artery plaques were detected as previously described. ${ }^{13}$ Briefly, each coronary segment was evaluated by placing the ROI in the artery lumen. The structure, which was attached to the coronary artery wall, and could be distinguished in at least 2 independent planes, was defined as the coronary artery plaque. The severity of stenosis was defined as $0 \%$ (0-24\%), 25\% (25-49\%), 50\% (50-74\%), 75\% (75-89\%), and $90 \%(90-99 \%)$ compared with the reference lumen, although these data were not used in this study because this classification was inconsistent with the purpose of this study. Plaque characteristics were defined by the CT value referenced from a previous report. ${ }^{14}$ Plaques with a $C T$ value $<50 \mathrm{HU}$ were defined as low-attenuation plaques, those with 51-150 HU were defined as intermediate-attenuation plaques, and those with $>151 \mathrm{HU}$ were defined as highattenuation plaques. To evaluate plaque volume, conterminous cross-sectional images ( $1 \mathrm{~mm}$ in thickness) of the coronary arteries taken with a fixed setting $(800 \mathrm{HU}$ window, 200 HU level) were used.

First, the target lesion was determined, and plaque areas were manually traced in the cross-sectional image. Second, the plaque volume of each CT value in the whole target lesion was calculated by the software. For accurate analysis, all coronary artery branches with a diameter $>1.5 \mathrm{~mm}$ were divided into 16 segments according to the American Heart Association classification. ${ }^{15}$ Finally, the calculated plaque volume for each segment was added up for each CT value. Total coronary plaque volume was calculated using the 


\begin{tabular}{|c|c|c|c|}
\hline & $\begin{array}{c}\text { MM } \\
(n=15)\end{array}$ & $\underset{(n=17)}{S}$ & $P$ value \\
\hline \multicolumn{4}{|l|}{ MSNA } \\
\hline Burst frequency (bursts/min) & $52.7 \pm 11.7$ & $57.9 \pm 12.3$ & 0.22 \\
\hline Burst incidence (bursts/100 heartbeats) & $77.3 \pm 14.9$ & $85.1 \pm 13.8$ & 0.14 \\
\hline Spike frequency (spikes/min) & $54.7 \pm 11.4$ & $62.8 \pm 12.9$ & 0.07 \\
\hline Spike incidence (spikes/100 heartbeats) & $80.9 \pm 11.3$ & $93.8 \pm 20.1$ & $<0.05$ \\
\hline \multicolumn{4}{|l|}{ Coronary artery plaque } \\
\hline Low-attenuation plaque volume $\left(\mathrm{mm}^{3}\right)$ & $202.8 \pm 175.5$ & $335.7 \pm 177.1$ & $<0.05$ \\
\hline Intermediate-attenuation plaque volume $\left(\mathrm{mm}^{3}\right)$ & $336.7 \pm 186.1$ & $518.6 \pm 289.8$ & $<0.05$ \\
\hline High-attenuation plaque volume $\left(\mathrm{mm}^{3}\right)$ & $440.6 \pm 146.0$ & $504.9 \pm 208.2$ & 0.16 \\
\hline Calcium score (points) & $120.3 \pm 307.8$ & $281.0 \pm 275.6$ & 0.29 \\
\hline RI & $1.02 \pm 0.29$ & $0.95 \pm 0.26$ & 0.47 \\
\hline
\end{tabular}

Values are mean \pm SD. MM, mild-moderate group; MSNA, muscle sympathetic nerve activity; RI, remodeling index; $\mathrm{S}$, severe group.

process shown in Figure 2.

To evaluate lesion vulnerability, positive remodeling was evaluated by calculating the remodeling index (RI) as previously described. ${ }^{\mathbf{1 6}}$ Briefly, RI was calculated by dividing the cross-sectional vessel wall area at the lesion of minimal lumen area by the reference area. The reference area was defined as the healthy non-plaque area that was as close as possible to the respective coronary lesion.

Calcium score was also calculated from non-contrast CT images using the Agatston score. ${ }^{17}$ In summary, a calcific lesion was defined as an area $\geq 1 \mathrm{~mm}^{2}$ above $130 \mathrm{HU}$, and a lesion score was determined according to the maximal CT number in the following manner: $1=130-199,2=200-299$, $3=300-399$, and $4 \geq 400 \mathrm{HU}$. Total calcium score was calculated by adding up each of these scores for all regions. Typical images of 320-slice CCT, coronary angiography (CAG), and intravascular ultrasonography (IVUS), performed subsequent to the CCT recording are shown in Figure 3A,B. The patient in Figure 3 had a significant stenosis in the left anterior descending coronary artery and then underwent CAG and IVUS-guided percutaneous coronary intervention. However, the patient's HR was $>65$ beats/min, CCT images were maintained and corresponded to both CAG and IVUS images.

\section{Statistical Analysis}

Continuous variables are presented as mean \pm standard deviation. All statistical analyses were performed using SPSS for Windows (version 17.0; SPSS Japan Inc., Tokyo, Japan). Univariate regression analysis was performed to evaluate correlations among each variable. Pearson's correlation analysis was used to assess the strength of the relationships between the variables. The unpaired Student's t-test was performed to compare differences between groups with homogeneous variances. If variance was heteroscedastic, Welch's t-test was used. Chi-squared test was adopted to compare differences of population rate between groups. $\mathrm{P}<0.05$ (two-sided) was considered significant.

According to previous studies, a sample size of 12 patients per group was estimated to provide $80 \%$ power to detect a $60 \mathrm{~mm}^{3}$ difference in mean plaque volume between groups, with $\alpha=0.05$. ${ }^{7,18}$

\section{Results}

In all, 214 patients were newly diagnosed with OSAS in the sleep disorder laboratory of Kanazawa Municipal Hospital between October 2013 and July 2016. After applying the inclusion and exclusion criteria, 112 patients underwent CCT measurement and of them, 65 did not undergo MSNA measurements, and 15 were excluded because of a low MSNA signal-to-noise ratio recording when evaluating single-unit MSNA. Finally, 32 patients were included in this study. Table 1 lists their baseline characteristics and medications. The study population consisted of $26(81 \%)$ men and $6(19 \%)$ women, with a mean age of 63.3 years, body mass index (BMI) of $26.3 \mathrm{~kg} / \mathrm{m}^{2}$, mean $\mathrm{HR}$ of 70.7 beats/min, mean LDL cholesterol of $119.3 \mathrm{mg} / \mathrm{dL}$, and mean $\mathrm{HbA} 1 \mathrm{c}$ of $5.9 \%$. Almost half of the participants had hypertension (44\%), 19\% had diabetes mellitus, and 25\% had dyslipidemia. Most of these participants with diseases had already received oral medication therapy. The mean AHI was 33.8 events $/ \mathrm{h}$, which is generally regarded as severe OSAS.

\section{Comparison of Patient Characteristics According to OSAS Severity}

The subjects were divided into 2 groups according to their AHI score. Patients with 5-29 events/h were assigned to the mild-moderate OSAS group (MM) and patients with $\geq 30$ events/h were assigned to the severe OSAS group (S). Table 2 lists the baseline characteristics and medications of each group. No differences were observed between the 2 groups in age, sex, BMI, blood pressure, HR, LDLcholesterol or HbAlc. Prevalence rates of hypertension, diabetes mellitus, dyslipidemia, and medications for these diseases also did not differ significantly. All of the measured PSG sleep parameters (AHI, 3\% oxygen desaturation index (ODI), arousal index) were significantly higher in the $\mathrm{S}$ group than in the MM group. Table 3 shows the comparison of MSNA and plaque characteristics for the 2 groups. Single-unit spike incidence was significantly higher in the $\mathrm{S}$ group than in the MM group, and other MSNA (burst frequency/incidence, spike frequency) also tended to be higher in the $\mathrm{S}$ group than in the MM group.

Regarding the comparison of coronary plaque volume, low- and intermediate-attenuation plaque volumes were 


\begin{tabular}{|c|c|c|c|c|c|c|c|c|c|c|}
\hline & \multicolumn{2}{|c|}{ Low } & \multicolumn{2}{|c|}{ Intermediate } & \multicolumn{2}{|c|}{ High } & \multicolumn{2}{|c|}{ Ca score } & \multicolumn{2}{|c|}{$\mathbf{R} \mathbf{I}$} \\
\hline & $\mathbf{r}$ & $P$ value & $\mathbf{r}$ & $P$ value & $\mathbf{r}$ & $P$ value & $\mathbf{r}$ & $P$ value & $\mathbf{r}$ & $P$ value \\
\hline Age (years) & 0.01 & 0.94 & 0.18 & 0.33 & 0.06 & 0.75 & 0.67 & $<0.05$ & 0.23 & 0.20 \\
\hline BMI $\left(\mathrm{kg} / \mathrm{m}^{2}\right)$ & 0.08 & 0.67 & 0.12 & 0.89 & 0.02 & 0.68 & 0.26 & 0.15 & 0.22 & 0.23 \\
\hline Systolic BP (mmHg) & 0.06 & 0.75 & 0.18 & 0.33 & 0.19 & 0.30 & 0.28 & 0.12 & 0.10 & 0.60 \\
\hline Diastolic BP (mmHg) & 0.03 & 0.86 & 0.04 & 0.84 & 0.02 & 0.90 & 0.28 & 0.12 & 0.23 & 0.20 \\
\hline Heart rate (beats/min) & 0.26 & 0.16 & 0.33 & 0.06 & 0.21 & 0.24 & 0.21 & 0.25 & 0.30 & 0.10 \\
\hline LDL-cholesterol (mg/dL) & 0.15 & 0.42 & 0.08 & 0.67 & 0.01 & 0.94 & 0.14 & 0.44 & 0.16 & 0.39 \\
\hline $\mathrm{HbA1c}(\%)$ & 0.22 & 0.23 & 0.09 & 0.63 & 0.002 & 0.99 & 0.32 & 0.08 & 0.10 & 0.60 \\
\hline Burst frequency (bursts/min) & 0.23 & 0.21 & 0.02 & 0.92 & 0.17 & 0.34 & 0.33 & 0.06 & 0.12 & 0.52 \\
\hline Burst incidence (bursts/100 heartbeats) & 0.25 & 0.17 & 0.03 & 0.89 & 0.17 & 0.37 & 0.22 & 0.22 & 0.10 & 0.60 \\
\hline Spike frequency (spikes/min) & 0.28 & 0.13 & 0.12 & 0.51 & 0.12 & 0.52 & 0.08 & 0.66 & 0.11 & 0.55 \\
\hline Spike incidence (spikes/100 heartbeats) & 0.28 & 0.12 & 0.18 & 0.33 & 0.05 & 0.79 & 0.03 & 0.87 & 0.15 & 0.41 \\
\hline $\mathrm{AHI}$ (events/h) & 0.52 & $<0.05$ & 0.33 & 0.07 & 0.24 & 0.18 & 0.02 & 0.90 & 0.26 & 0.15 \\
\hline Arousal index (events/h) & 0.18 & 0.44 & 0.13 & 0.11 & 0.12 & 0.50 & 0.01 & 0.94 & 0.01 & 0.95 \\
\hline $3 \%$ ODI (events/h) & 0.32 & 0.08 & 0.20 & 0.18 & 0.21 & 0.24 & 0.12 & 0.52 & 0.19 & 0.30 \\
\hline
\end{tabular}

$\mathrm{Ca}$, calcium; High, high-attenuation plaque volume; Intermediate, intermediate-attenuation plaque volume; Low, low-attenuation plaque volume. Other abbreviations as in Tables 1,3.

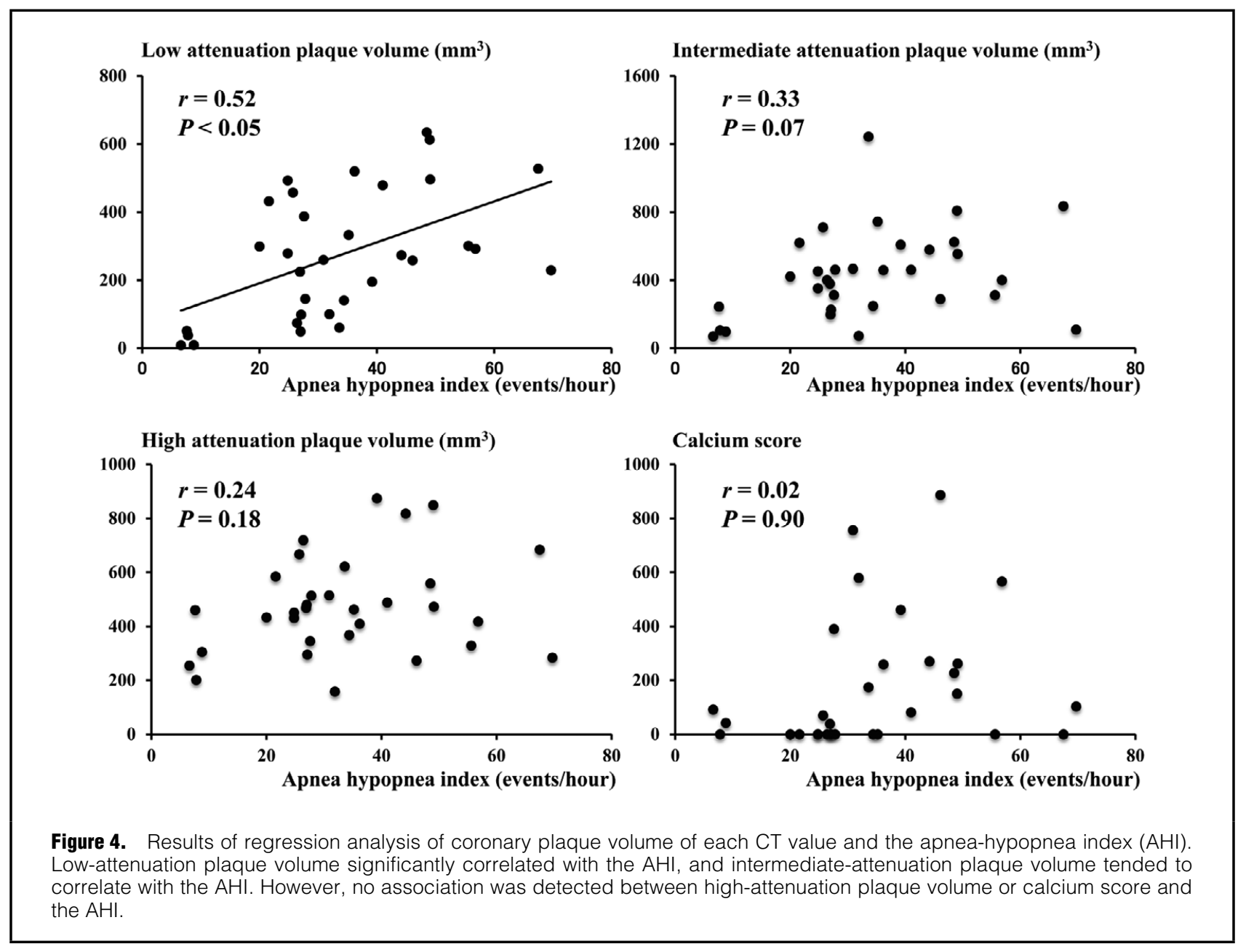


significantly higher in the $\mathrm{S}$ group than in the MM group; however, high-attenuation plaque volume, calcium score and RI did not differ significantly between the groups.

\section{Relationship Between Coronary Plaque Characteristics and Each Parameter}

Table 4 lists the results of regression analysis of coronary plaque characteristics and other clinical parameters including MSNA and sleep parameters. No associations were observed between coronary plaque volume of any CT value or index (calcium score and RI) and other parameters except AHI; however, calcium score was significantly associated with age. In regard to the AHI, low-attenuation plaque volume was significantly associated with the AHI $(\mathrm{r}=0.52$, $\mathrm{P}<0.05$ ), and intermediate-attenuation plaque volume tended to correlate with the $\mathrm{AHI}$; however, high-attenuation plaque volume, and calcium score did not (Figure 4).

\section{Discussion}

This is the first report to evaluate the relationship between the coronary plaque volume of each CT value measured by 320-slice CCT and OSAS severity with regard to PSG parameters or daytime SNA, using single- and multi-unit MSNA, in patients with OSAS. The high AHI group exhibited significant low-attenuation plaque volume, calcium score and daytime MSNA compared with the moderate AHI group. There were no differences in blood pressure, BMI, LDL-cholesterol, HbAlc, or prevalence of diseases (hypertension, dyslipidemia and diabetes mellitus), which are regarded as confounding risk factors for CVD progression, between the 2 groups. In the regression analysis, a significant association was observed only between lowattenuation plaque volume and AHI. No association was observed between the coronary plaque volume of any CT value and daytime MSNA.

\section{0-Slice CCT}

The characteristic of 320-slice CCT is large z-axis detector coverage of $16 \mathrm{~cm}$, allowing for acquisition of the whole heart within 1 heartbeat using a non-helical volumetric scanning approach. As a result, 320-slice CCT eliminates the presence of stair-step artifacts and typical pitch artifacts, which are often a problem in patients with high or irregular HR evaluated using conventional CCT measurements. ${ }^{8}$ Although the superiority of the diagnostic performance of 320 -slice CCT has not been established, it is reported to have a comparable level of diagnostic accuracy for detecting significant coronary artery stenosis as CAG in patients with a HR $>64$ beats/min or HR irregularities, as well as in patients with a HR $<65$ beats/min. ${ }^{12}$ Another report demonstrated high image quality of 320 -slice CCT in atrial fibrillation patients, almost equal to that in normal sinus rhythm patients; ${ }^{19}$ conventional CCT evaluation, such as with 64-slice, is not recommended for patients with arrhythmias according to recent CCT guidelines. ${ }^{20}$

Patients with OSAS present with a high HR, not only during apnea events but also during the daytime because of the increased cardiac sympathetic drive and altered cardiovascular variability. ${ }^{21}$ In our study, the resting HR was 70.7 beats $/ \mathrm{min}$, which is not considered to be within the applicable range for 64-slice CCT. The HRs of 22 of the 32 patients examined in this study were $\geq 65$ beats/min during the CCT measurement, even after administration of a shortacting adrenergic $\beta-1$ blocker. However, temporal resolution of the CCT images was preserved in these patients, comparable to CAG and IVUS recordings (Figure 3).

\section{Coronary Artery Plaque Characteristics in OSAS Patients}

Plaque volume and characteristics are well-known contributors to CVD development.22,23 Two previous studies have shown a significant association between $\mathrm{AHI}$ and the mean non-calcified coronary plaque volume, as evaluated by CCT, in OSAS patients. ${ }^{7,24}$ However, both studies used 64-slice CCT, and did not report a detailed association between the AHI and coronary plaque characteristics with each CT value. In addition, one of those previous reports used cardiorespiratory polygraphy instead of $\mathrm{PSG},{ }^{7}$ which may have underestimated the actual AHI.

We used the gold standard PSG (i.e., 320-slice CCT), and found a detailed association between coronary plaque volume in each CT value and the AHI. A significant association between the AHI and calcium score has been reported previously. ${ }^{25}$ However, in our study, no linear correlation was observed between the AHI and calcium score. Calcium score was only significantly correlated with age. These results indicated that coronary calcification may be induced by the duration of morbidity of sleep apnea in each patient, not the temporal increase in disease severity. Unfortunately, we could not clarify the actual duration of sleep apnea in each patient, but it is believed that older patients would have suffered from sleep apnea for a longer period. According to our results and previous reports, the large, non-calcified, low-attenuation plaque volume may represent a characteristic of the coronary artery in severe OSAS patients, rather than aging-related calcification.

\section{Low-Attenuation Plaque Volume and the AHI}

A low CT value in CCT images is considered to be 1 of the specific features of vulnerable plaque..$^{26}$ In OSAS patients, intermittent hypoxia and hypercapnia during apnea events cause systemic inflammation, a rapid increase in blood pressure, and oxidative stress, which lead to vascular endothelial dysfunction and alteration of the shear stress on the arteries. ${ }^{27,28}$ These reactions during sleep apnea may directly contribute to vulnerable plaque proliferation. ${ }^{29}$ In addition, daytime hypertension, dyslipidemia and insulin resistance, which are led by sleep apnea, are believed to indirectly induce atherosclerosis in OSAS patients. ${ }^{28,30}$ In this study, the coronary low-attenuation plaque volume, as assessed by 320 -slice CCT, was associated with the AHI, reflecting the actual magnitude of OSAS severity, but not with $3 \%$ ODI or the arousal index. These results indicated the importance of the $\mathrm{AHI}$ as a therapeutic target for inhibiting CVD progression in patients with OSAS. However, further studies are warranted to evaluate whether a reduction in the AHI would decrease vulnerable plaque volume. Positive remodeling is also an important predictor of acute coronary events, ${ }^{31}$ but no association was observed between RI and AHI in this study. Positive remodeling is generally formed subsequent to plaque proliferation, and is related to plaque area. ${ }^{32}$ Many participants in this study had never experienced CVD, indicating that they were in the early stages of atherosclerosis. Among these patients, plaque proliferation beginning prior to remodeling may have been a more sensitive parameter than vascular vessel remodeling for detecting coronary artery vulnerable sign.

\section{SNA and CVD in OSAS Patients}

A recent animal study reported a significant relationship 
between augmented SNA and vulnerable plaque proliferation. Increased central sympathetic drive stimulated hematopoietic cell activation via the $\beta 3$ adrenergic receptor, resulting in accelerated neutrophil and monocyte production. Extensive release of inflammatory leukocytes promoted plaque inflammation and vulnerability. ${ }^{6}$ In this study, high AHI patients showed significantly high daytime MSNA compared with moderate AHI patients. However, contrary to our hypothesis, there was no association between the coronary plaque volume of any $\mathrm{CT}$ value and daytime MSNA. In our study, daytime MSNA did not correlate with coronary plaque volume. Augmented SNA in OSAS patients is believed to carry over into the daytime; however, SNA is more prominent during the apnea phase. A previous report demonstrated unidirectional antegrade and retrograde oscillated brachial artery flow, and observed rapidly augmented MSNA during apnea events in a patient with OSAS, suggesting that vasoconstrictive effects induced by elevated SNA impose significant oscillatory shear stress on arteries. ${ }^{33}$ Accordingly, increased SNA induced by apnea during the night may be more likely to promote vulnerable plaque proliferation compared with daytime SNA.

\section{Study Limitations}

First, the sample size was relatively small. However, despite the small study population, a significant association was detected between AHI and low-attenuation plaque volume. Second, plaque characteristics and vulnerability were evaluated only by $\mathrm{CT}$ value. Other characteristics, such as spotty calcification and napkin-ring sign of the coronary artery, were not assessed because of quantification difficulties. However, the plaque characteristics assessed by CT value have been established as reliable markers of plaque vulnerability. ${ }^{31}$ Additionally, the prognostic value of lowand intermediate-plaque volume was recently reported in patients with coronary artery disease. ${ }^{34}$ Third, as mentioned previously, MSNA was not assessed during the nighttime, because the patient's leg movement easily removes the nerve-recording electrode during sleep.

\section{Conclusions}

In this study, the AHI score was significantly associated with coronary low-attenuation plaque volume. Daytime MSNA, including single-unit MSNA, had no association with the coronary plaque volume of any of the $\mathrm{CT}$ values. These results indicated that the AHI plays a critical role in developing CVD in patients with OSAS by accelerating vulnerable plaque proliferation.

\section{References}

1. Mooe T, Rabben T, Wiklund U, Franklin KA, Eriksson P. Sleep-disordered breathing in men with coronary artery disease. Chest 1996; 109: 659-663.

2. Shah NA, Klar Yaggi H, Concato J, Mohsenin V. Obstructive sleep apnea as a risk factor for coronary events or cardiovascular death. Sleep Breath 2010; 14: 131-136.

3. Hamaoka T, Murai H, Kaneko S, Usui S, Okabe Y, Tokuhisa $\mathrm{H}$, et al. Single-unit muscle sympathetic nerve activity reflects sleep apnea severity, especially in severe obstructive sleep apnea patients. Front Physiol 2016; 7: 1-9.

4. Trombetta IC, Somers VK, Maki-Nunes C, Drager LF, ToschiDias E, Alves MJNN, et al. Consequences of comorbid sleep apnea in the metabolic syndrome: Implications for cardiovascular risk. Sleep 2010; 33: 1193-1199.

5. Kimmerly DS, Morris BL, Floras JS. Apnea-induced cortical BOLD-fMRI and peripheral sympathoneural firing response patterns of awake healthy humans. PLoS One 2013; 8: 20-23.
6. Heidt T, Sager HB, Courties G, Dutta P, Iwamoto Y, Zaltsman A, et al. Chronic variable stress activates hematopoietic stem cells. Nat Med 2014; 20: 754-758.

7. Kent BD, Garvey JF, Ryan S, Nolan G, Dodd JD, McNicholas WT. Severity of obstructive sleep apnoea predicts coronary artery plaque burden: A coronary computed tomographic angiography study. Eur Respir J 2013; 42: 1263-1270.

8. Li S, Ni Q, Wu H, Peng L, Dong R, Chen L, et al. Diagnostic accuracy of 320-slice computed tomography angiography for detection of coronary artery stenosis: Meta-analysis. Int J Cardiol 2013; 168: 2699-2705.

9. Uehara M, Funabashi N, Takaoka H, Komuro I. Quality of coronary arterial 320-slice computed tomography images compared with 16-slice computed tomography images in subjects with chronic atrial fibrillation. Int $J$ Cardiol 2011; 149: e90e93.

10. Hunt SA, Abraham WT, Chin MH, Feldman AM, Francis GS, Ganiats TG, et al. 2009 Focused Update Incorporated Into the ACC/AHA 2005 Guidelines for the Diagnosis and Management of Heart Failure in Adults. J Am Coll Cardiol 2009; 53: e1e90.

11. Chesson AL, Berry RB, Pack A. Practice parameters for the use of portable monitoring devices in the investigation of suspected obstructive sleep apnea in adults. Sleep 2003; 26: 907-913.

12. Uehara M, Takaoka H, Kobayashi Y, Funabashi N. Diagnostic accuracy of 320-slice computed-tomography for detection of significant coronary artery stenosis in patients with various heart rates and heart rhythms compared with conventional coronaryangiography. Int J Cardiol 2013; 167: 809-815.

13. Achenbach S, Moselewski F, Ropers D, Ferencik M, Hoffmann U, MacNeill B, et al. Detection of calcified and noncalcified coronary atherosclerotic plaque by contrast-enhanced, submillimeter multidetector spiral computed tomography: A segmentbased comparison with intravascular ultrasound. Circulation 2004; 109: 14-17.

14. Voros S, Rinehart S, Qian Z, Joshi P, Vazquez G, Fischer C, et al. Coronary atherosclerosis imaging by coronary $\mathrm{CT}$ angiography: Current status, correlation with intravascular interrogation and meta-analysis. JACC Cardiovasc Imaging 2011; 4: 537-548.

15. Austen WG, Edwards JE, Frye RL, Gensini GG, Gott VL, Griffith LS, et al. A reporting system on patients evaluated for coronary artery disease. Circulation 1975; 51: 5-40.

16. Kröner ESJ, Van Velzen JE, Boogers MJ, Siebelink HMJ, Schalij MJ, Kroft LJ, et al. Positive remodeling on coronary computed tomography as a marker for plaque vulnerability on virtual histology intravascular ultrasound. Am J Cardiol 2011; 107: $1725-1729$.

17. Agatston AS, Janowitz WR, Hildner FJ, Zusmer NR, Viamonte M, Detrano R. Quantification of coronary artery calcium using ultrafast computed tomography. J Am Coll Cardiol 1990; 15: $827-832$.

18. Turmel J, Sériès F, Boulet LP, Poirier P, Tardif JC, RodésCabeau J, et al. Relationship between atherosclerosis and the sleep apnea syndrome: An intravascular ultrasound study. Int $J$ Cardiol 2009; 132: 203-209.

19. Uehara M, Funabashi N, Ueda M, Murayama T, Takaoka H, Sawada K, et al. Quality of coronary arterial 320-slice computed tomography images in subjects with chronic atrial fibrillation compared with normal sinus rhythm. Int J Cardiol 2011; 150: $65-70$.

20. Taylor AJ, Cerqueira M, Hodgson JM, Mark D, Min J, O'Gara $\mathrm{P}$, et al. ACCF/SCCT/ACR/AHA/ASE/ASNC/NASCI/SCAI/ SCMR 2010 Appropriate Use Criteria for Cardiac Computed Tomography. J Am Coll Cardiol 2010; 56: 1864-1894.

21. Narkiewicz K, Montano N, Cogliati C, van de Borne PJH, Dyken ME, Somers VK. Altered cardiovascular variability in obstructive sleep apnea. Circulation 1998; 98: 1071-1077.

22. Motoyama S, Sarai M, Harigaya H, Anno H, Inoue K, Hara T, et al. Computed tomographic angiography characteristics of atherosclerotic plaques subsequently resulting in acute coronary syndrome. J Am Coll Cardiol 2009; 54: 49-57.

23. Versteylen MO, Kietselaer BL, Dagnelie PC, Joosen IA, Dedic A, Raaijmakers RH, et al. Additive value of semiautomated quantification of coronary artery disease using cardiac computed tomographic angiography to predict future acute coronary syndrome. J Am Coll Cardiol 2013; 61: 2296-2305.

24. Sharma S, Gebregziabher M, Parker AT, Abro JA, Armstrong AM, Schoepf UJ. Independent association between obstructive sleep apnea and noncalcified coronary plaque demonstrated by noninvasive coronary computed tomography angiography. Clin 
Cardiol 2012; 35: 641-645.

25. Sorajja D, Gami AS, Somers VK, Behrenbeck TR, GarciaTouchard A, Lopez-Jimenez F. Independent association between obstructive sleep apnea and subclinical coronary artery disease. Chest 2008; 133: 927-933.

26. Rodriguez-Granillo GA, Carrascosa P, Bruining N, Waksman R, Garcia-Garcia HM. Defining the non-vulnerable and vulnerable patients with computed tomography coronary angiography: Evaluation of atherosclerotic plaque burden and composition. Eur Heart J Cardiovasc Imaging 2016; 17: 481 -491.

27. Meier-Ewert HK, Ridker PM, Rifai N, Regan MM, Price NJ, Dinges DF, et al. Effect of sleep loss on C-reactive protein, an inflammatory marker of cardiovascular risk. J Am Coll Cardiol 2004; 43: 678-683.

28. Somers VK, White DP, Amin R, Abraham WT, Costa F, Culebras A, et al. Sleep apnea and cardiovascular disease: An American Heart Association/American College of Cardiology Foundation Scientific Statement From the American Heart Association Council for High Blood Pressure Research Professional Education Committee, Council on Clinical Cardiology, Stroke Council, and Council On Cardiovascular Nursing: In collaboration with the National Heart, Lung, and Blood Institute
National Center on Sleep Disorders Research (National Institutes of Health). Circulation 2008; 118: 1080-1111.

29. Toutouzas K, Benetos G, Karanasos A, Chatzizisis YS, Giannopoulos AA, Tousoulis D. Vulnerable plaque imaging: Updates on new pathobiological mechanisms. Eur Heart $J$ 2015; 36: $3147-3154$.

30. Pamidi S, Tasali E. Obstructive sleep apnea and type 2 diabetes: Is there a link? Front Neurol 2012; 3: 1-13.

31. Motoyama S, Kondo T, Sarai M, Sugiura A, Harigaya H, Sato T, et al. Multislice computed tomographic characteristics of coronary lesions in acute coronary syndromes. $\mathrm{J} \mathrm{Am} \mathrm{Coll} \mathrm{Cardiol}$ 2007; 50: 319-326

32. Glagov S, Weisenberg E, Zarins CK, Stankunavicius R, Kolettis GJ. Compensatory enlargement of human atherosclerotic coronary arteries. $N$ Engl J Med 1987; 316: 1371-1375.

33. Millar PJ. Obstructive sleep apnea: A novel mechanism for endothelial dysfunction? Hypertension 2011; 58: e17-e18.

34. Dwivedi G, Liu Y, Tewari S, Inacio J, Pelletier-Galarneau M, Chow BJW. Incremental prognostic value of quantified vulnerable plaque by cardiac computed tomography: A pilot study. J Thorac Imaging 2016; 31: 373-379. 